\title{
CURVAS DE ABSORCIÓN DE NUTRIENTES EN PAPAYA (Carica papaya L.) CV. "POCOCÍ" EN LAS FASES DE CRECIMIENTO VEGETATIVO, FLORACIÓN E INICIO DE COSECHA
}

\author{
Róger Fallas $^{1 / *}$, Floria Bertsch ${ }^{*}$, Mauricio Barrientos ${ }^{* *}$ \\ Palabras clave: Carica papaya; fertilización; absorción de nutrientes; macronutrientes; micronutrientes; \\ acumulación de materia seca. \\ Keywords: Carica papaya; nutrient absorption; fertilization; macronutrients; micronutrients; dry matter \\ accumulation.
}

Recibido: 13/02/14

\section{RESUMEN}

Durante el 2010, en una plantación comercial de papaya cv. "Pococí", ubicada en Guácimo, Costa Rica, se determinó la curva de crecimiento del cultivo y las curvas de absorción de nutrimentos, mediante muestreos destructivos de plantas realizados desde el trasplante a partir de febrero del 2010 hasta 9 meses después del trasplante. Fueron obtenidas las curvas de absorción de nutrientes para un híbrido de alto rendimiento, así como sus requerimientos nutrimentales. Con una densidad de siembra de 1600 plantas por hectárea, se estimó que el híbrido "Pococí" requirió para su desarrollo hasta los 9 meses aproximadamente $354 \mathrm{~kg} \cdot \mathrm{ha}^{-1}$ de N, $101 \mathrm{~kg} \cdot \mathrm{ha}^{-1}$ de $\mathrm{P}_{2} \mathrm{O}_{5}, 498 \mathrm{~kg} \cdot \mathrm{ha}^{-1}$ de $\mathrm{K}_{2} \mathrm{O}, 174 \mathrm{~kg} \cdot \mathrm{ha}^{-1}$ de $\mathrm{CaO}$, 106 kg.ha- ${ }^{-1}$ de $\mathrm{MgO}$ y $40 \mathrm{~kg} \cdot \mathrm{ha}^{-1}$ de S, cantidades requeridas que, en su mayoría, superan a las que normalmente están siendo aplicadas mediante los programas de fertilización en el país.

\footnotetext{
1 Autor para correspondencia. Correo electrónico: rogerarmando.fallas@ucr.ac.cr

* Centro de Investigaciones Agronómicas, Universidad de Costa Rica.
}

Aceptado: 30/06/14

** Centro Agrícola Cantonal de Guácimo. 


\section{INTRODUCCIÓN}

La papaya es una planta originaria de Mesoamérica, con centro de diversificación en América Central y el sur de México (Campostrini y Glenn 2007), razón por la cual en Costa Rica es posible encontrar una gran variabilidad genética de este cultivo.

Esa gran variabilidad representa un gran potencial para el desarrollo de nuevas variedades o híbridos, como lo es el caso del híbrido Pococí desarrollado en Costa Rica por parte del personal del MAG-INTA y la Universidad de Costa Rica.

El desarrollo del híbrido "Pococí", ha venido a solucionar problemas de producción y comercialización, ya que entre sus características destacan sus altos rendimientos, la calidad de fruta, uniformidad en las plantaciones y fruta que llega al mercado, además de buen sabor y aroma, lo cual ha propiciado un incremento en la demanda del mercado interno y ha favorecido la exportación de este híbrido hacia otros países como Canadá, Estados Unidos, Bélgica y otros países (CNP 2012).

Con el desarrollo del híbrido se ha tratado de establecer un paquete tecnológico que favorezca a los productores que están involucrados en la actividad en Costa Rica, la mayoría de ellos pequeños productores, según lo demuestran estudios relacionados sobre control de malezas (Bogantes et ál. 2010a), uso de coberturas (Bogantes y Mora 2004) y sexado (Saalau et ál. 2009), etc.

Por otra parte, la información referente a la demanda de nutrientes por el cultivo de la papaya es escaza y específicamente para el híbrido Pococí, no ha sido registrada, información que puede ser de utilidad para realizar programas de fertilización adecuados a las necesidades de este híbrido y que podrían servir como punto de partida para la fertilización de papaya en general.

En Costa Rica la fertilización realizada al híbrido normalmente se basa en la experiencia adquirida por productores con varios años en la actividad o en otros casos se adapta la información de curvas de absorción desarrolladas en otros países, con material genético diverso y de rendimientos inferiores a los presentados por los híbridos modernos como Pococí.

Otra metodología que ha sido adoptada para el cálculo de las necesidades nutrimentales de las plantaciones, es mediante el uso de los datos de extracción de nutrientes por la fruta (Bogantes et ál. 2010b), con la limitante que este método no contempla las necesidades nutrimentales de la plantación para el desarrollo de estructuras vegetativas de la raíz, el tallo y las hojas, por lo que se podrían subestimar las cantidades de nutrientes necesarias para alcanzar el mayor potencial productivo de estos híbridos modernos.

Estudios recientes han demostrado que la planta de papaya requiere de altas aplicaciones de nutrientes para lograr su óptima productividad (Kirad et ál. 2010, Kumar et ál. 2010), lo cual coincide con la característica de alta productividad de los híbridos de papaya modernos.

En 1980 en Brasil, Cunha y Haag determinaron las curvas de absorción para un material de papaya que alcanzó rendimientos de 30 t.ha ${ }^{-1}$, para el caso del híbrido Pococí en Costa Rica han sido reportados rendimientos a nivel experimental superiores a las 130 t.ha $^{-1} \mathrm{y}$ a nivel comercial cerca de 80 t.ha $^{-1}$ (Bogantes y Mora 2006, CNP 2012).

$\mathrm{Si}$ se consideran como referencia esas diferencias en el potencial productivo de los híbridos modernos, podrían suponerse demandas de nutrientes superiores en híbridos como Pococí. Un indicio de esta mayor absorción y demanda de nutrientes por los híbridos modernos es relatado por Oliveira y Caldas (2004), quienes en el estado de Bahía, Brasil, al utilizar un híbrido con un rendimiento estimado de 93 t.ha $^{-1} \cdot a_{n} o^{-1}$ (rendimiento similar al obtenido por el híbrido Pococí), obtuvieron el máximo rendimiento económico del cultivar "Sunrise Solo" cuando fueron utilizadas dosis de $347 \mathrm{~kg}$ de $\mathrm{N}$ y $360 \mathrm{~kg}$ de $\mathrm{K}_{2} \mathrm{O}$ por hectárea, sin embargo como mencionado para el caso de Pococí no han sido determinadas sus necesidades nutrimentales.

Otros estudios desarrollados en la India también concuerdan con una alta demanda de nutrientes por el cultivo, por ejemplo Kumar et ál. (2010) determinaron que la dosis de $\mathrm{K}$ en que se 
maximizaba el rendimiento se encontraba alrededor de los 300 g.planta ${ }^{-1}$.año ${ }^{-1}$ de $\mathrm{K}$, con una dosis estandarizada de N y P de 300 g.planta ${ }^{-1}$.año ${ }^{-1}$. Otro aspecto mencionado en el estudio de Kumar et ál. (2010) es la respuesta diferencial entre cultivares de papaya frente a diferentes dosis de $\mathrm{K}$, en su estudio uno de los cultivares evaluados presentó respuesta a la adición de $\mathrm{K}$ en valores cercanos a los 450 g.planta ${ }^{-1} \cdot$ año $^{-1} \mathrm{~K}$, cuando se utilizó una dosis de $\mathrm{N}$ de 750 g.planta ${ }^{-1}$.año ${ }^{-1}$.

También, en diversos estudios se ha determinado que uno de los elementos que más impacta sobre el rendimiento de la papaya es el N (Marinho et ál. 2001, Oliveira y Caldas 2004, Rajbhar et ál. 2010), elemento que cuando es aplicado en altas dosis en sistemas agrícolas ha demostrado una baja eficiencia de aprovechamiento en otros cultivos (Colla et ál. 2011), producto de las diferentes pérdidas que puede sufrir este nutriente en los sistemas de producción.

Esa alta demanda relatada anteriormente, junto con las posibles pérdidas que pueden sufrir los nutrientes cuando son aplicados como fertilizantes, hacen necesario el entendimiento de las necesidades de absorción de nutrientes en el cultivo, para aplicar así las cantidades de nutrientes necesarias en las etapas en que se requieren, y lograr la sostenibilidad económica y ambiental del cultivo.

El objetivo de este trabajo fue establecer las curvas de absorción de nutrientes del híbrido de papaya "Pococí" en una plantación comercial, durante las etapas que comprenden desde la siembra hasta los 9 meses posteriores al establecimiento.

\section{MATERIALES Y MÉTODOS}

Durante el 2010, en una plantación comercial de papaya cv. "Pococí", ubicada en Guácimo, Costa Rica, fue determinada durante 9 meses la producción de materia seca de las diferentes partes de la planta (raíz, tallo, hojas y estructuras reproductivas (flores y frutos)), con muestreos destructivos de plantas completas. En dichos tejidos fue determinada la concentración de nutrientes para cada una de las épocas evaluadas, mediante el secado de la muestra, digestión nitríco-perclórica en horno de microondas y lectura en ICP-OES de los diferentes elementos.

Mediante los datos de materia seca y concentración de nutrientes en los diferentes tejidos de la planta, fue calculada la absorción total de N, P, K, Ca, Mg, S, B y Zn en cada tejido, y en la planta (total). Esto mismo se realizó para el $\mathrm{Fe}$, $\mathrm{Mn}$, e $\mathrm{Cu}$ sin embargo en esta publicación no se muestran los resultados. Con el promedio de esos datos fueron estimadas las curvas de absorción de nutrientes.

A lo largo del ciclo del cultivo fueron colectadas 3 plantas por cada época de muestreo representativas del buen desarrollo de las mismas dentro de la plantación. Para esto entre las plantas con mejor desarrollo, se seleccionaron al azar 3 plantas, fueron extraídas completas y se seccionaron en los diferentes tejidos para su respectivo análisis. La plantación presentó un buen desarrollo y estado nutrimental durante el período evaluado.

El suelo sobre el que fue desarrollada la plantación presentó inicialmente las siguientes características químicas (Cuadro 1).

Cuadro 1. Características químicas del suelo base del experimento para determinación de las curvas de absorción de nutrientes en papaya cv. Pococí.

\begin{tabular}{|c|c|c|c|c|c|c|c|c|c|}
\hline $\mathrm{pH}$ & $\mathrm{Al}^{*}$ & $\mathrm{Ca}^{*}$ & $\mathrm{Mg}^{*}$ & $\mathrm{~K}^{* *}$ & $\mathrm{P}^{* *}$ & $\mathrm{Zn}^{* *}$ & $\mathrm{Cu}^{* *}$ & $\mathrm{Fe}^{* *}$ & $\mathrm{Mn}^{* *}$ \\
\hline & \multicolumn{4}{|c|}{$\operatorname{cmol}(+) .1^{-1}$} & \multicolumn{5}{|c|}{ mg.dm ${ }^{-3}$} \\
\hline 5,4 & 0,28 & 4,32 & 0,77 & 0,18 & 5,5 & 1,7 & 4 & 105 & 7 \\
\hline
\end{tabular}

*Determinado mediante solución extractora $\mathrm{KCl} 1$ mol. ${ }^{-1}$.

** Determinado mediante solución extractora Olsen modificada (Díaz-Romeu y Hunter 1982). 


\section{RESULTADOS Y DISCUSIÓN}

\section{Absorción total}

En concordancia con su alto rendimiento, el híbrido presentó una alta demanda de nutrientes (Cuadro 2).

El K se presentó como el nutriente de mayor demanda por el cultivo, seguido por el $\mathrm{N}$, lo cual contrasta con lo reportado por Cunha y Haag (1980) que en Brasil encontraron al N como el nutriente de mayor absorción en el cultivo.

En diversas regiones del mundo ha sido reconocida la respuesta a la adición del $\mathrm{N}$ y $\mathrm{K}$ en el cultivo de papaya, por ejemplo en la India, Kumar et ál. (2010) encontraron influencia de la fertilización potásica sobre factores de rendimiento como el número de frutas por planta y el peso de fruta, Rajbhar et ál. (2010) también encontraron la mayor cantidad de frutos cuando utilizaron dosis altas de K (250 g.planta ${ }^{-1}$ de K) e intermedias de $\mathrm{N}$ $\left(250\right.$ g.planta $\left.{ }^{-1}\right)$, mientras que el mayor peso de frutos lo obtuvieron con la combinación de las dosis más altas de $\mathrm{N}$ y $\mathrm{K}$ evaluadas.

Esta alta demanda de $\mathrm{K}$ encontrada para el híbrido Pococí, podría ser de importancia para brindar un mejor manejo de este nutriente en las plantaciones comerciales, ya que como presentan Bogantes et ál. (2010), la absorción estimada a partir de la extracción por fruta $\left(302 \mathrm{~kg} \cdot \mathrm{ha}^{-1}\right.$ $\mathrm{K}_{2} \mathrm{O}$ ), cantidad que es utilizada para el cálculo de las dosis recomendadas en los programas de fertilización en Costa Rica, podrían no ser suficientes para satisfacer las demandas reales del cultivo (498 kg.ha- ${ }^{-1} \mathrm{~K}_{2} \mathrm{O}$ según este estudio (Cuadro 2)).

Cuadro 2. Absorción nutrimental máxima total de una plantación de papaya híbrido Pococí, durante el período vegetativo hasta el inicio de cosecha ( 9 meses después del trasplante) en Guácimo, Costa Rica*.

\begin{tabular}{ccccccc}
\hline $\mathrm{N}$ & $\mathrm{P}_{2} \mathrm{O}_{5}$ & $\mathrm{~K}_{2} \mathrm{O}$ & & $\mathrm{CaO}$ & $\mathrm{MgO}$ & $\mathrm{S}$ \\
\hline \multirow{2}{*}{221,2} & & & g.planta $^{-1}$ & & & \\
& 63,1 & 311,4 & & 109 & 66,5 & 25 \\
353,9 & & & kg.ha $^{-{ }^{*}}$ & & & \\
& 101 & 498,3 & & 174,4 & 106,3 & 40 \\
\hline
\end{tabular}

*Absorción estimada con una densidad de siembra de 1600 plantas.ha ${ }^{-1}$.

Otra posible implicación del uso de los datos de únicamente la extracción por la fruta como método de dosificación de fertilizantes en el cultivo, podría ser un empobrecimiento gradual de los suelos, principalmente porque en el país como medida fitosanitaria se acostumbra remover todas las plantas y restos del cultivo una vez finalizado el ciclo de producción, básicamente para reducir inóculo de enfermedades y plagas que puedan afectar a plantaciones futuras y como puede ser observado en el apartado sobre curvas de absorción, una cantidad importante de nutrientes se encuentra en las estructuras vegetativas (raíz, tallos y hojas), por lo que al retirar este material se estarían extrayendo también nutrimentos del sistema que no son contabilizados mediante el método de dosificación basado en la extracción por la fruta.

Para el caso del $\mathrm{N}$ las cantidades requeridas por el cultivo fueron altas (221 g.planta $\left.{ }^{-1}\right)$ (Cuadro 2), inclusive superiores en más de un $100 \%$ de lo reportado en otros estudios como por ejemplo Cunha y Haag (1980), quienes luego de conducir una plantación durante más de un año encontraron una absorción de $\mathrm{N}$ inferior a 70 g.planta ${ }^{-1}$. 
Esta alta demanda de $\mathrm{N}$ por el cultivo debe ser manejada con precaución para no ocasionar problemas de acidificación del suelo, principalmente si son utilizadas fuentes con alto índice de acidificación. Cabe destacar que en la región Atlántica de Costa Rica donde se desarrolló este estudio, el régimen de precipitación es bastante alto, por lo que tienden a ser utilizadas fuentes amoniacales para disminuir las pérdidas de $\mathrm{N}$ por lixiviación, razón por la cual se debe prestar especial atención a las áreas de producción a través del tiempo para verificar y controlar los posibles problemas de acidificación. Así, la determinación de las necesidades de $\mathrm{N}$ por el híbrido de papaya brinda información importante para dar un mejor manejo, tanto en términos nutrimentales de la papaya, como en términos de la conservación de la fertilidad de suelos y el manejo de los problemas de acidez en el cultivo.

Semejante a lo encontrado por Cunha y Haag (1980) y lo mencionado por Mesquita et ál. (2010) el Ca se presentó como el tercer elemento de mayor demanda en el cultivo; a pesar de esta similitud, las cantidades de Ca absorbidas por el híbrido Pococí fueron muy superiores que lo reportado por Cunha y Haag (1980). Dicha demanda de Ca podría estar relacionada con la obtención de fruta de buena calidad, ya que este elemento ha sido relacionado con la calidad poscosecha de la fruta (Qiu et ál. 1995). Al igual que el $\mathrm{Ca}$, las cantidades de $\mathrm{Mg}$ y S absorbidas por el híbrido Pococí en este estudio, fueron muy superiores a las reportadas por Cunha y Haag (1980) en Brasil, presentándose según su

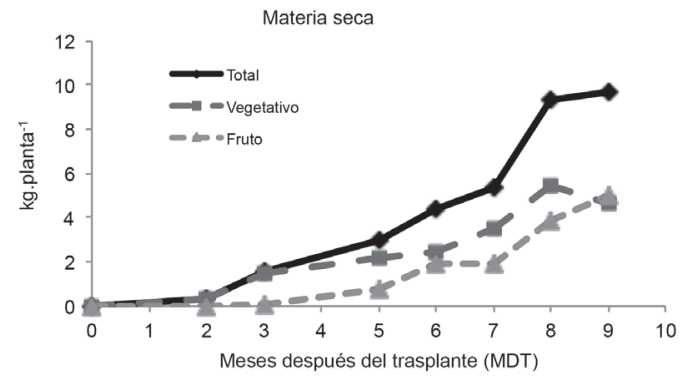

importancia (una mayor absorción) en el siguiente orden $\mathrm{Ca}, \mathrm{Mg}, \mathrm{P}$ y $\mathrm{S}$ respectivamente.

\section{Curvas de absorción}

\section{Materia seca}

El desarrollo del híbrido de papaya presentó un pequeño acúmulo de materia seca durante las primeras etapas posteriores a la siembra.

En la etapa posterior a los 2 meses después de la siembra (MDT), el híbrido presentó el primer pico de crecimiento, como se observa en la Figura 1 , en esa etapa experimentó un incremento en peso del $10 \%$ en relación con la materia seca total máxima acumulada durante el período de evaluación ( 9 meses en campo). Posteriormente en la fase que comprende de los 3 a los 5 meses de desarrollo, el híbrido obtuvo aumentos del $16 \%$ de la materia seca en relación con el máximo alcanzado.

Luego el híbrido Pococí incrementó su materia seca a una tasa superior al $11 \%$ mensual y en la fase que comprende de los 7 a los 8 meses experimentó el mayor acúmulo de materia seca (Figura 1), por lo que esta etapa podría corresponder también a la de mayor demanda de nutrientes por el cultivo durante el desarrollo vegetativo y la etapa de inicio de cosecha.

Este gran aumento en el acúmulo de materia seca parece estar relacionado con el incremento en el tamaño y peso de los frutos, tal y como se demuestra mediante el incremento en el peso seco de fruta, pero también acompañado de aumentos en el peso seco de la planta (Figura 1).

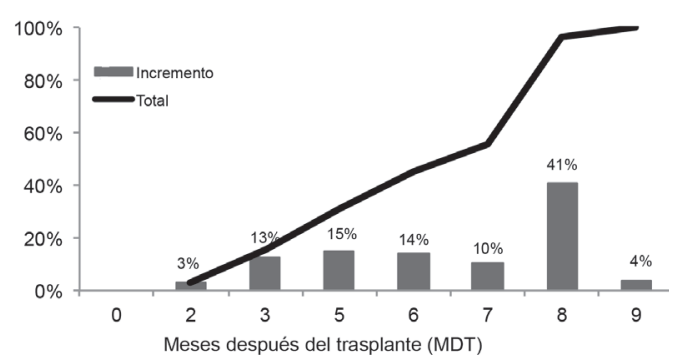

Fig. 1. Acúmulo de materia seca por una plantación de papaya (Carica papaya) híbrido Pococí durante el crecimiento vegetativo hasta el inicio de cosecha (9 meses después del trasplante). 
En la etapa de los 9 MDT no se reportaron incrementos importantes en la producción de materia seca, esta situación se relaciona con la fase de cosecha de frutos, la cual puede haber influido en la reducción de la materia seca de la parte vegetativa de la planta mediante translocación de asimilados desde estos órganos hacia los frutos. Además, otro factor importante de mencionar es que en este estudio no pudo ser contabilizada la cantidad de fruta extraída entre los 8 y 9 MDT, correspondiente al inicio de cosecha, por lo cual el dato de la absorción total acumulada por la fruta para esa etapa podría ser mayor que la presentada en este estudio, el dato de los 9 MDT no fue excluido específicamente para denotar que ocurren salidas del sistema por medio de la fruta, las cuales deben ser evaluadas en otros estudios posteriores.

\section{Nitrógeno, Fósforo y Potasio}

Para el caso del $\mathrm{N}$ en la etapa que comprende de los 2 a los 5 MDT, el híbrido de papaya absorbió una gran cantidad del elemento (Figura 2), seguido por un período con absorciones menores al 10\% mensual hasta alcanzar los 7 MDT.

En general para N, P y K, la etapa de los 7 a los 8 meses fue la de mayor absorción, lo cual concuerda con el acúmulo de materia seca de planta (crecimiento vegetativo) y el aumento en el peso de los frutos (Figura 1), con este comportamiento en términos relativos (absorción porcentual del nutriente) más notorio para $\mathrm{N}$ y $\mathrm{P}$. Es importante mencionar al respecto que la papaya no detiene su crecimiento vegetativo durante la fase de fructificación, emite hojas nuevas aun cuando se trata de formar y desarrollar frutos, razón por la cual debe de prestarse especial atención a la nutrición del cultivo en estas etapas.

Para el caso del K la mayor absorción del nutriente difirió respecto a lo presentado por el $\mathrm{N}$ y el P, básicamente en que para la etapa de los 6 a los 7 MDT también se presentó una importante absorción de K (27\% del total), la cual inclusive es similar o comparable a la absorción que se presentó en la etapa de los 7 a los 8 meses. 

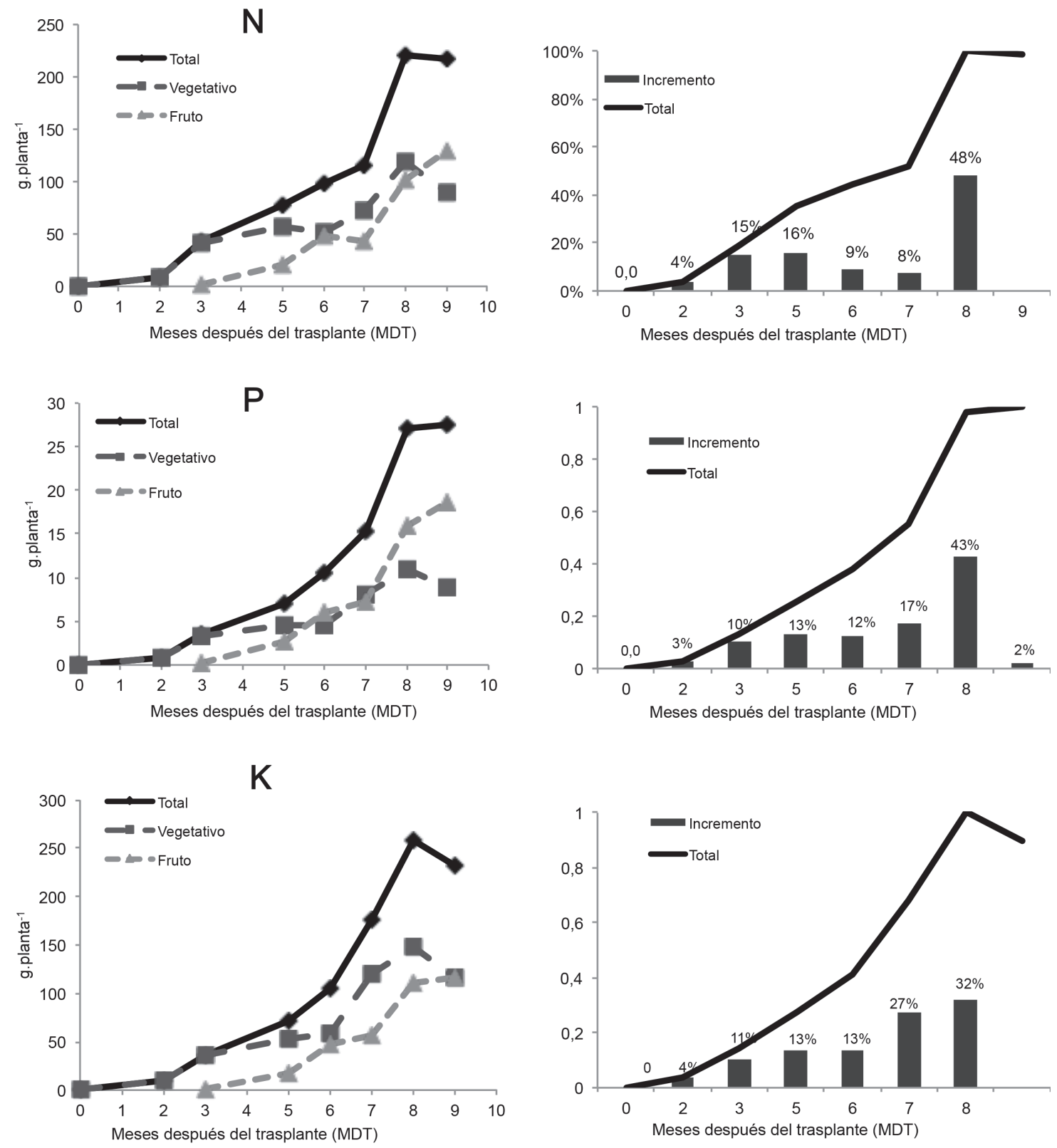

Fig. 2. Curvas de absorción de N, P y K en el cultivo de papaya (Carica papaya) cv. Pococí. 


\section{Calcio, Magnesio y Azufre}

El Ca como es presentado en la Figura 3 mostró 2 etapas de acúmulo del nutriente, la primera etapa se ubica entre los 2 y 5 meses y correspondió a una absorción del 30\% del total de $\mathrm{Ca}$ acumulado durante los 9 meses que duró el experimento, posteriormente entre el quinto y sexto mes parece haber un cese en el acúmulo (únicamente acumuló 3\%) y luego el cultivo tiende a absorber importantes cantidades del nutriente, como se muestra en la Figura 3, probablemente relacionado también a la demanda de Ca por el fruto.

En el caso del $\mathrm{Mg}$ se presentaron absorciones continuas del nutriente desde los primeros meses, ya que mostró una importante absorción en el período entre los 2 y 3 MDT (Figura 3). Al igual que ocurrió con el Ca y demás nutrientes ya relatados el período que abarca entre 7 y 8 meses presentó la mayor absorción del elemento $(\mathrm{Mg})$, relacionado con la demanda de nutrientes para la fructificación.

El S se diferenció de los otros nutrimentos en que la absorción del nutriente por parte de la fruta superó a la absorción o demanda del nutriente que presentó el tejido vegetativo (raíz, tallo, pecíolo y limbos de hojas), esto señala que debe prestarse especial atención a las aplicaciones de este elemento en las etapas de desarrollo del fruto. La etapa que abarcó de los 7 a los 8 meses se presentó una absorción de casi el 50\% del total absorbido por la planta, también relacionado al proceso de fructificación. 

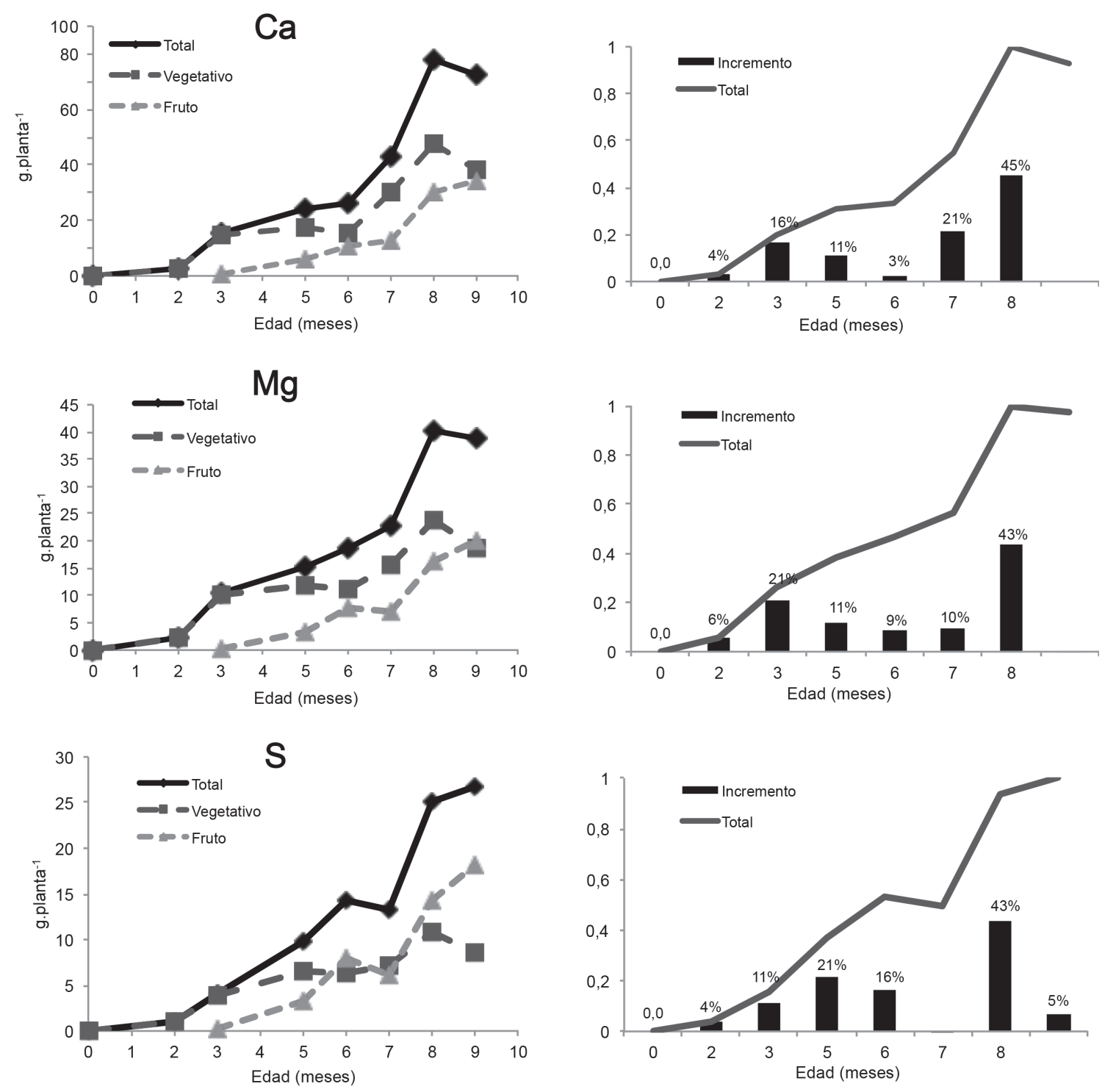

Fig. 3. Curvas de absorción de Ca, Mg y S en papaya (Carica papaya) cv. Pococí. 


\section{Zinc y Boro}

El Zn se presentó como un nutrimento sin un comportamiento de absorción definido, situándose las etapas de mayor requerimiento entre los 6 y 9 meses.

El B se absorbió en cantidades importantes (aproximadamente 32\%) hasta antes de los 3 meses, y presentó otro pico de absorción entre los 7 y 8 MDT (etapa de formación de frutos e inicio de cosecha). Singh et ál. (2005) reportan efectos positivos de la aplicación de B junto con Zn sobre el rendimiento de la papaya, elementos que en Costa Rica han sido considerados como limitantes en una gran cantidad de suelos. La
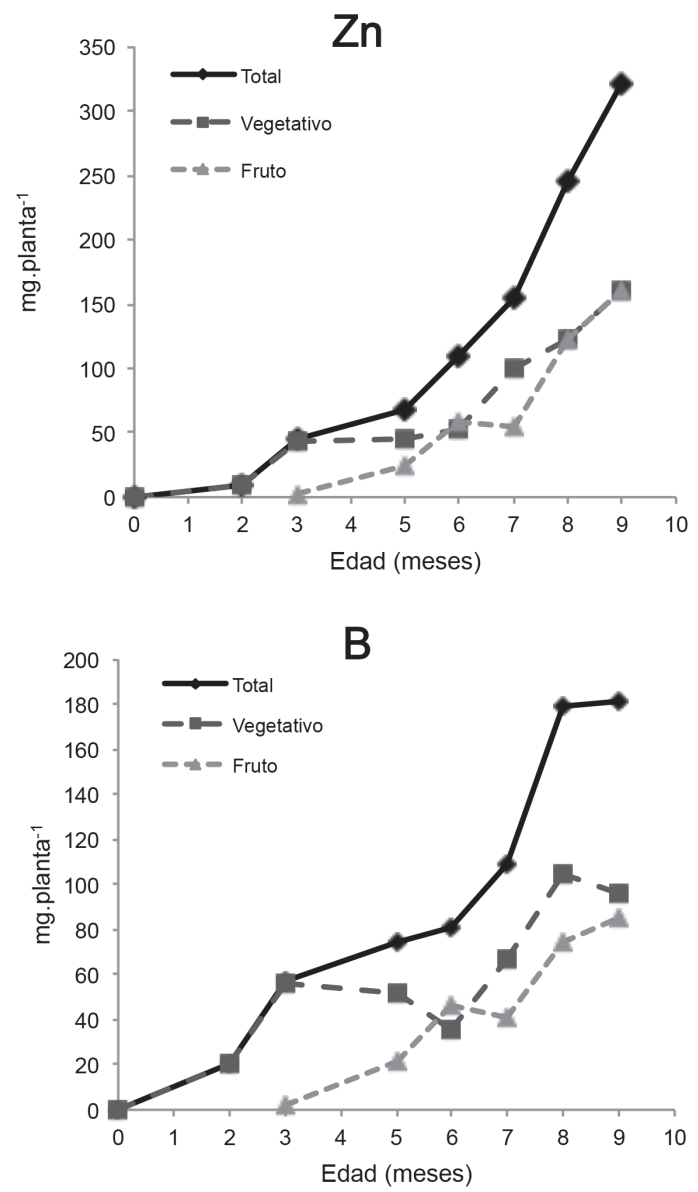

deficiencia de B en el cultivo de la papaya ha sido relatada como muy problemática por una gran cantidad de investigadores (Wang y Ko 1975, Nautiyal et ál. 1986, Nishina 1991, Nelson 2012), presentándose con problemas de deformación de la fruta.

Dada la importancia de este nutrimento (B) para el cultivo y la común ocurrencia de problemas de deficiencia, las aplicaciones de este elemento probablemente deban realizarse antes de los 3 MDT y en las épocas de mayor crecimiento y formación de la fruta (en el caso de este experimento a partir de los 6 MDT), según lo presentado en la Figura 4.
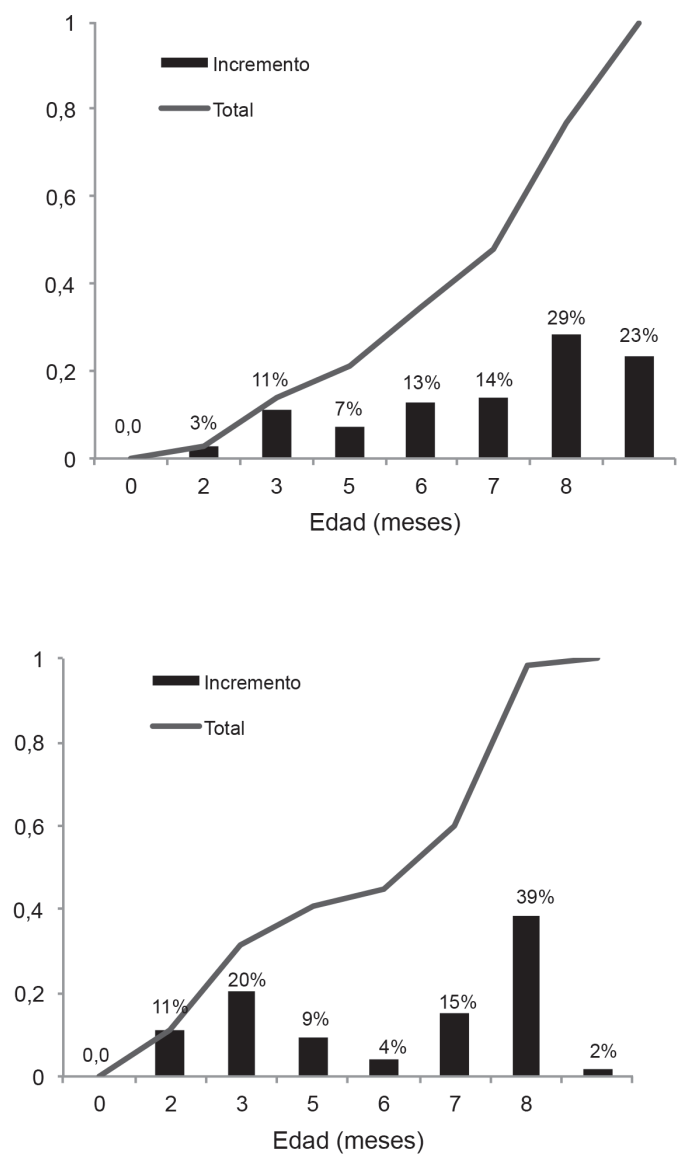

Fig. 4. Curvas de absorción de Zn y B en papaya (Carica papaya) cv. Pococí. 


\section{CONCLUSIONES}

El $\mathrm{K}$ es el elemento de mayor demanda en la papaya cv. Pococí.

La edad entre 7 y 8 MDT es la etapa de mayor demanda de nutrientes por parte del híbrido Pococí según el período evaluado en este estudio.

La absorción total de nutrientes por parte del híbrido podría ser mayor a las cantidades que actualmente se adicionan vía fertilización, lo cual podría ocasionar un empobrecimiento gradual de los suelos sometidos a la producción de papaya, conforme se realicen siembras sucesivas del cultivo.

Es posible que aún con las altas producciones encontradas actualmente con el híbrido Pococí (130 t.ha-1), no se alcance su máximo potencial de rendimiento, ya que con fertilizaciones que aportaban cantidades mucho menores de los requerimientos reales del cultivo, ha sido alcanzado ese alto nivel de producción (hasta 130 t.ha ${ }^{-1}$ ), probablemente un aporte de nutrientes más acorde con las necesidades reales de la planta podrían reflejarse en aumentos del rendimiento del híbrido, situación que algunos productores señalan haber observado, pero que requiere de un proceso de validación con investigaciones futuras.

\section{LITERATURA CITADA}

BOGANTES A., HERNÁNDEZ M., MORA E. 2010a. Herbicidas para el control de Spermacoce tenuior L. en papaya (Carica papaya). Agronomía Mesoamericana 21(1):185-192.

BOGANTES A., MORA E. 2004. Factibilidad técnica del uso de cobertura vegetal en papaya (Carica papaya L.) mediante la aplicación localizada de herbicida. Agronomía Mesoamericana 15(2):193-199.

BOGANTES A., MORA E. 2006. Validación del rendimiento del híbrido de papaya "Pococí" (Carica papaya) en el Caribe de Costa Rica. Revista Alcances Tecnológicos 4(1):53-58.

BOGANTES A., MORA E., UMAÑA G., LORÍA C. 2010b. Guía para la producción de la papaya en Costa Rica. Consultado: 25/06/2013. Disponible en http://www. eefb.ucr.ac.cr/images/Publicidad/Guia\%20para\%20 la $\% 20$ produccion $\% 20$ de $\% 20$ papaya $\% 20$ en $\% 20$ Costa\%20Rica.pdf
CAMPOSTRINI E., GLENN D. 2007. Ecophysiology of papaya: a review. Braz. J. of Plant Physiol. 19(4):413424.

CNP 2012. Análisis de Mercados Papaya. Consultado: 30/07/2013. Disponible en http://www.simacr.go.cr/ index.php/papaya

COLLA G., ROUPHAEL Y., MIRABELLI C., CARDARELLI M. 2011. Nitrogen-use efficiency traits of mini-watermelon in response to grafting and nitrogen-fertilization doses. Journal of Plant Nutrition and Soil Science 174:933-941.

CUNHA R., HAAG H. 1980. Nutrição mineral do mamoeiro (Carica papaya L.). V- Marcha de absorção de nutrientes em condições de campo. Anais da Escola Superior de Agricultura "Luiz de Queiroz" 37:631668.

DÍAZ-ROMEU R., HUNTER A. 1982. Metodología de muestreo de suelos, análisis químico de suelos y tejido vegetal y de investigaciones en invernadero. CATIE Turrialba. $61 \mathrm{p}$.

KIRAD K., BARCHE S., SINGH D. 2010. Integrated Nutrient Management in papaya (Carica papaya L.) cv. Surya. Acta Horticulturae 851:377-380.

KUMAR N., SOORIANATHASUNDARAM K., MEENAKSHI N., MANIVANNAN M., SURESH J., NOSOV V. 2010. Balanced fertilization in papaya (Carica papaya L.) for higher yield and quality. (Proc. 2nd IS on Papaya) Acta Horticulturae 851:357-362.

MARINHO C., OLIVEIRA M., MONNERAT P., VIANNI R., MALDONADO J. 2001. Fontes e doses de nitrogênio e a qualidade dos frutos do mamoeiro. Scientia Agricola 58(2):345-348

MESQUITA E., CAVALCANTE L., GONDIM S., CAMPOS V., CAVALCANTE I., GONDIM P. 2010. Teores foliares e exportação de nutrientes do mamoeiro Baixinho de Santa Amália tratado com biofertilizantes. Pesq. Agropec. Trop. Goiânia 40(1):66-76.

NAUTIYAL B., SHARMA C., AGARWALA S. 1986. Iron zinc and boron deficiency in papaya. Scientia Horticulturae 1-2:115-123.

NELSON S. 2012. Boron deficiency of papaya. College of Tropical Agriculture and Human Resources. University of Hawaii at Manoa. Plant disease PD-91. Consultado: 29/07/2013. Disponible en http://www. ctahr.hawaii.edu/oc/freepubs/pdf/PD-91.pdf

NISHINA M. 1991. Bumpy fruit of papaya as related to boron deficiency. Hawaii Inst. of Trop. Agric. And Human Resources, Commodity Fact Sheet PA-4 (B), Univ. of Hawaii. Consultado: 29/07/2013. Disponible en http://www.ctahr.hawaii.edu/oc/freepubs/pdf/CFSPA-4B.pdf

OLIVEIRA A., CALDAS R. 2004. Produção do mamoeiro em função de adubação com nitrogênio, fósforo 
e potássio. Revista Brasileira de Fruticultura 26(1):160-163.

QIU Y., NISHINA M., PAULL R. 1995. Papaya fruit growth, calcium uptake, and fruit ripening. J. Amer. Soc. Hort. Sci. 120(2):246-253.

RAJBHAR P., SINGH G., LAL M. 2010. Effect of N, P, K and spacing on growth and yield of papaya (Carica papaya L.) cv. Pant Papaya 1. (Proc. $2^{\text {nd }}$ IS on Papaya) Acta Horticulturae 851:425-428.
SAALAU E., BARRANTES W., LORÍA C., BRENES A., GÓMEZ L. 2009. Identificación mediante PCR del sexo de la papaya (Carica papaya L.), híbrido "Pococí". Agronomía Mesoamericana 20(2):311-317.

SINGH D., PAUL P., GHOSH S. 2005. Response of papaya to foliar application of boron, zinc and their combinations. Research on Crops 6(2):277-280.

WANG D., KO W. 1975. Relationship between deformedfruit disease of papaya and boron deficiency. Phytopathology 65:445-447. 